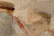

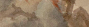

Fis.

Mos:

(1)

ars $\rightarrow$

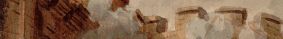

WDithe te is
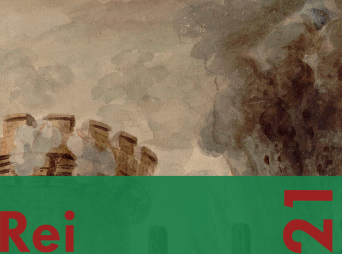

Revista digital de Historia

y Didáctica de la Historia 
Revista anual

Fecha de inicio: 1995

RevistaPantaRei.pantarei@um.es

\section{Edita:}

Centro de Estudios del Próximo Oriente y la

Antigüedad Tardía - CEPOAT

Edificio Universitario Saavedra Fajardo.

Universidad de Murcia

C/ Actor Isidoro Máiquez, 9

30007 - MURCIA - ESPAÑA

Teléfono: $(+34) 868883890$

cepoat@um.es

Web: https://revistas.um.es/pantarei

\section{Ediciones de la Universidad de Murcia - EDITUM}

Edificio Pleiades. Campus de Espinardo.

Universidad de Murcia

C/ Campus, s/n

30100 - MURCIA - ESPAÑA

Teléfono: (+34) 868883013

editum@um.es

Web: https://www.um.es/web/editum/

En portada: Prise de la Bastille. Biblioteca Nacional de Francia (BNF).

Edición 2021

ISSNe: 2386-8864

ISSN: $1136-2464$

Depósito legal: MU-966-1995

Responsables de los textos: sus autores.

Responsable de la presente edición: Consejo Editorial de Panta Rei. 


\section{CONSEJO DE REDACCIÓN}

Coordinador editorial

Egea Vivancos, Alejandro [Didáctica de la Historia, Universidad de Murcia]

Secretaria

Arias Ferrer, Laura [Didáctica de la Historia, Universidad de Murcia]

Editores

Bellatti, Ilaria [Didáctica de la Historia, Universidad de Barcelona]

Jiménez Vialás, Helena [Historia Antigua, Universidad de Murcia]

Martínez Gil, Tània [Didáctica de Historia,

Universidad de Barcelona]

Meseguer Gil, Antonio José [Historiador, Profesor de Secundaria]

Ortiz García, Jónatan [Arqueología, Universidad de Alcalá de Henares]

Romero Molero, Alberto [Arqueología, Universidad Isabel I]

Sáez Giménez, David Omar [Historiador, Profesor de Secundaria]

Sáez Rosenkranz, Isidora V. [Didáctica de la Historia, Universidad de Barcelona]

Sánchez Mondejar, Celso Miguel [Arqueólogo,

Patrimonio Inteligente]

Responsables de traducción y corrección lingüística

Martínez Martínez, Cristina [Profesora de Secundaria, Sociedad Española de Lenguas Modernas] Albaladejo Albaladejo, Sara [ISEN-Universidad de Murcia]

\section{CONSEJO ASESOR}

Adroher Auroux, Andrés María [Arqueología, Universidad de Granada]

Albero Muñoz, $M^{a}$ del Mar $\left[H{ }^{a}\right.$ del Arte, Universidad de Murcia]

Alia Miranda, Francisco [Historia Contemporánea, UCLM]

Arciniega García, Luis [Historia del Arte, Universidad de Valencia]

Barrio Barrio, Juan Antonio [Historia Medieval,

Universidad de Alicante]

Castellano i Solé, Núria [Egiptología, Schola

Didàctica Activa S.L.]

Chapman, Arthur [History Education, University

College of London, Reino Unido]

Chavarria Arnau, Alejandra [Arqueología, Università di Padova, Italia]

Cid López, Rosa María [Historia Antigua, Universidad de Oviedo]

Cobacho López, Ángel [Derecho, Universidad de Murcia]

Cuenca López, José María [Didáctica de la Historia, Universidad de Huelva]

Egea Bruno, Pedro M. ${ }^{a}$ [Historia Contemporánea, Universidad de Murcia]
Feijoo Martínez, Santiago [Arqueología, Consorcio

Ciudad Monumental de Mérida]

García Atienzar, Gabriel [Prehistoria, Universidad de

Alicante]

Ginestí Rosell, Anna [Filología Clásica, Katholische

Universität Eichstätt-Ingolstadt]

González Monfort, Neus [Didáctica de la

Historia, Universidad Autónoma de Barcelona]

González Soutelo, Silvia [Arqueología, Universidad

Autónoma de Madrid]

Haber Uriarte, María [Prehistoria, Universidad de

Murcia]

Hernández de la Fuente, David [Filología Clásica,

Universidad Complutense]

Hutson, Scott R. [Anthropology, University of Kentucky, EEUU]

lgual Luis, David [Historia Medieval, UCLM]

Irigoyen López, Antonio [Historia Moderna,

Universidad de Murcia]

Jover Maestre, Francisco Javier [Prehistoria,

Universidad de Alicante]

Mahony, Simon [Digital Humanities, University College

of London, Reino Unido]

Marsilla de Pascual, Francisco Reyes [Técnicas

historiográficas, Universidad de Murcia]

Martínez-Burgos García, Palma [H. ${ }^{a}$ del Arte, UCLM]

Mathis, Christian [Didaktik der Geschichte, PH Zürich]

Miralles Maldonado, José Carlos [Filología Clásica,

Universidad de Murcia]

Molina Gómez, José Antonio [Historia Antigua,

Universidad de Murcia]

Mónica Ghirardi [Historia Moderna, Universidad

Nacional de Córdoba, Argentina]

Navarro Espinach, Germán [Historia Medieval,

Universidad de Zaragoza]

Ni Cassaithe, Caitriona [Didáctica de la Historia,

Dublin City University, Irlanda]

Noguera Celdrán, José Miguel [Arqueología,

Universidad de Murcia]

Ortiz Heras, Manuel [Historia Contemporánea, UCLM]

Panzram, Sabine [Historia Antigua, Universität

Hamburg]

Pérez Molina, Miquel Emilio [Filología Clásica,

Universidad de Murcia]

Pinto, Helena [Didáctica de la Historia, CITCEM

University of Porto, Portugal]

Prados Martínez, Fernando [Arqueología,

Universidad de Alicante]

Sánchez lbáñez, Raquel [Didáctica de la Historia, Universidad de Murcia]

Sancho Gómez, Miguel Pablo [Educación, UCAM]

Victoria Moreno, Diego [Historia Contemporánea, UNED]

Vilar García, María José [Historia Contemporánea,

Universidad de Murcia]

Vivas Sainz, Inmaculada [H. ${ }^{a}$ del Arte, UNED]

Zamora López, José Ángel [Próximo Oriente Antiguo, CCHS-CSIC] 



\section{Índice}

\section{Artículos}

Libelos, camarillas y banquetes: una propuesta para reconstruir las proclamaciones militares en la 7 Antigüedad Tardía a través de los casos de Probo, Magnencio y Juliano

Miguel Pablo Sancho Gómez

El uso de los videojuegos para la enseñanza de la Historia Antigua en Educación Secundaria: una investigación educativa

Manuel Jesús Jaldón-Méndez Sánchez

'What did it achieve?' - Students' conceptions about the significance of the French Revolution

Christian Mathis

Pervivencia e influencia de los mitos fundacionales del franquismo en los textos escolares de la enseñanza secundaria castellanoleonesa

Sergio Ibáñez Llorente y Almudena Alonso-Centeno

La enseñanza y aprendizaje de las Ciencias Sociales a través del patrimonio, videojuegos y emociones. 103 Estudio de caso en un IES de Huelva (España)

Rocío Jiménez-Palacios y José María Cuenca López

Desarrollo de la conciencia histórica. Una propuesta de intervención y evaluación para la Educación de Adultos

Héctor López-Bajo, Rosendo Martínez Rodríguez y María Sánchez-Agustí

Concepciones de docentes en formación suecos y españoles sobre la desigualdad de género en perspectiva temporal

Jorge Ortuño Molina y Fredrik Alvén

Narrativa y significación histórica en la comunicación de los museos nacionales canadienses en Facebook

Iñaki Navarro-Neri, Pilar Rivero, Borja Aso y Ana Mendioroz-Lacambra

\section{Entrevista}

Haciendo historia para el bien común. Entrevista a Linda S. Levstik

Laura Arias Ferrer y Alejandro Egea Vivancos

\section{Reseñas}

M. Sartre (2021), Le Bateau de Palmyre. Quand les mondes anciens se rencontraient (Vle siècle av. J.- 239

C./Vle siècle ap. J.-C.), Paris: Éditions Tallandier

Juan Álvarez García

Janire Castrillo, Las mujeres vascas durante la Baja Edad Media. Vida familiar, capacidades jurídicas, 245 roles sociales y trabajo, Madrid, Sílex Universidad, 2020

Iratxe Gillate

M. Ortiz Heras (coord.). (Manual de) Historia de España (siglo XX). Del desastre del 98 a la crisis 249 sistémica actual, Albacete: Altabán, 418 págs. ISBN: 978-84-15252-46-7

Herminio Lebrero Izquierdo 



\title{
El uso de los videojuegos para la enseñanza de la Historia Antigua en Educación Secundaria: una investigación educativa
}

\section{The use of video games for the teaching of Ancient History in Secondary Education: an educational investigation}

\author{
Manuel Jesús Jaldón-Méndez Sánchez \\ Universidad de Huelva \\ manueljaldon@colegiodiocesano.net \\ (iD) $\underline{0000-0002-7166-9161}$
}

Recibido: $27 / 02 / 2021$

Aceptado: 17/09/2021

\section{Resumen}

El objetivo de este trabajo es valorar la idoneidad del videojuego Dominations como herramienta para crear un aprendizaje significativo en el alumnado de $1 .^{\circ}$ de ESO para los contenidos relacionados con la cultura mesopotámica, así como los beneficios que aporta su utilización en el aula. Para ello, se ha diseñado una investigación de tipo interpretativa estructurada en diferentes fases (búsqueda de experiencias de aula, preparación y diseño de los instrumentos de investigación y didáctica, investigación en el aula y evaluación de los resultados), teniendo como herramienta principal el videojuego. Este proceso revela, además de la mejora de la motivación en el aula, una mejor percepción de los contenidos, así como una mejor comprensión del tiempo histórico. El videojuego supone pues una excelente herramienta dentro del marco educativo formal para la enseñanza y el aprendizaje de la civilización mesopotámica.

\section{Palabras clave}

Investigación, videojuego, Historia, Enseñanza, Abstract
The objective of this work is to analyse the
suitability of the Dominations video game as a
tool to create meaningful learning in 1 st year
ESO students for the content related to
Mesopotamian culture, as well as the benefits
that its use in the classroom provides. To do this,
an interpretive-type research structured in
different phases has been designed (search
for classroom experiences, preparation and
design of research and didactic instruments,
research in the classroom and evaluation of the
results), with the video game as the main tool.
This process reveals, in addition to improved
motivation in the classroom, a better
perception of the content, as well as a better
understanding of historical time. Thus, the
video game is an excellent tool within the
formal educational framework for the teaching
and learning of the Mesopotamian civilization.

\section{Keywords}

Research, Videogames, History, Teaching, Ancient History 


\section{Introducción}

Los modelos de enseñanza y aprendizaje que se utilizan actualmente han cambiado muy poco a lo largo de los años, aún más cuando hablamos de la enseñanza de la historia, pues todavía predomina el modelo tradicional memorístico-repetitivo, donde mayoritariamente se establece un tipo de comunicación: la unidireccional del profesorado hacia el alumnado (Estepa, 2017). En años recientes se viene investigando acerca de nuevos métodos de enseñanza en esta materia dentro del contexto formal. Entre ellos, son numerosos los estudios que se han publicado acerca de la enseñanza y el aprendizaje de la Historia, que incluyen nuevos elementos tecnológicos y audiovisuales, como las webquests, blogs, stopmotion o m-learning (Ibañez-Etxeberria et al., $2011)$.

El uso de los videojuegos como herramienta educativa en el ámbito de las Ciencias Sociales ha sido estudiado en España en los últimos años (Egea et al., 2017, Iturriaga, 2015; JiménezPalacios, 2020; Martín-Cáceres y Cuenca López, 2019). En esta ocasión, se pretende explorar las posibilidades de un videojuego de carácter lúdico. En concreto, se analizará el potencial que posee el videojuego denominado Dominations. A través del estudio de este caso, se comprobará si es posible trabajar el sentido social de la historia, los procesos de evolución histórica, la distinción de periodos, así como la experimentación de los cambios que se producen en las sociedades, el conocimiento, la valoración del patrimonio y el medio ambiente.

Dicho recurso quedó integrado dentro de una unidad didáctica que ha sido conjuntamente evaluada con la aplicación del videojuego mediante un método de investigación cualitativa en el marco del paradigma interpretativo. Para ello se hizo uso de un cuestionario enfocado en el contenido impartido a través del videojuego y secuencia creada, así como de la observación participante. Se llevó a cabo en un aula de $1 .^{\circ}$ de ESO de un centro concertado perteneciente a la periferia de la ciudad de Huelva y en el que confluyen menores provenientes de toda la ciudad $y$, en concreto, de los barrios colindantes. La finalidad última era conocer el impacto educativo que puede tener el uso de un videojuego en la enseñanza de los contenidos relacionados con la Prehistoria y Mesopotamia.

\section{Marco Teórico}

En la actualidad, la sociedad se articula por el uso de las nuevas tecnologías, y estas van ligadas a un aprendizaje que se incorporan dentro de un entorno digital que está presente en todo momento y que es imprescindible su conocimiento. Esta cuestión no puede olvidarse a la hora de hablar de la educación, ya que, gracias a esto, el profesorado ha ampliado sus horizontes a través de la introducción de metodologías innovadoras en el aula. Es en este ámbito donde hay que trabajar, ya que el uso de las nuevas tecnologías es, en muchos casos, insuficiente $y$, en ocasiones, inadecuado. Son por ello necesarios unos conocimientos sobre estas que permitan al profesorado su integración en el aula, de ahí que se hable de los videojuegos para enseñar. Su uso, en general, es minoritario y anecdótico en el aula (Martín-Cáceres y Cuenca López, 2019), puesto que suponen una entrada directa a las nuevas tecnologías y que, a pesar de ser criticados, no usarlos implica desaprovechar una potente herramienta educativa (Gros, 2000).

El juego es una característica de la especie humana (Del Moral, 1996), por ello, diferentes pedagogos han hablado de la función del juego en la educación. Algunos afirman que cuanto 
más se juegue durante las horas de aprendizaje, mejor será este (Siurot, 1964), así como que la experiencia de jugar a cualquier juego siempre conlleva un aprendizaje (Becker, 2011). Hasta finales del siglo XIX, jugar había estado relacionado con el entretenimiento y la diversión, concepto que, gracias a la Escuela Nueva, cambió, adquiriendo un importante protagonismo como metodología de enseñanza. El juego pasó a ser una herramienta con un gran potencial educativo que desarrollaba múltiples capacidades en el alumnado (Gros, 2000). Además, supone una forma de involucrar al alumnado en el proceso educativo (Egea-Vivancos y Arias-Ferrer, 2020). La didáctica de la Historia se encuentra en un punto entre tradición e innovación en la escuela del siglo XXI y eso supone un problema, sobre todo en lo relacionado con la enseñanza y el aprendizaje de la Historia, pues se necesitan herramientas para captar la motivación del alumnado y poder generar procesos educativos satisfactorios. Entre estos elementos de motivación se encuentran los videojuegos, pues favorecen, entre otros aspectos, el desarrollo de la capacidad investigadora y del pensamiento histórico (Luna et al., 2019).

Hablar de videojuegos es hablar de un producto cultural que se caracteriza por pertenecer a la cultura popular (Barbier, 2014) y que está creciendo año tras año desde el 2007 (54\% del consumo audiovisual) (Anderson et al., 2008). Estas cifras van en aumento, puesto que, en el año 2019, España estuvo entre los diez primeros países en empleo de videojuegos, siendo las consolas de sobremesa el dispositivo más utilizado para jugar. Se estima que unos 15 millones de personas entre los 6 y 64 años son consumidores de videojuegos. También el uso por parte de las mujeres ha sido un $42 \%$ mayor el último año. Se establece así que el consumo medio de la población española es de unas 7,5 horas semanales (AEVI, 2020).

Los viodeojuegos constituyen uno de los objetos preferidos de la juventud y prácticamente están presentes en casi todos los hogares. De este modo, es necesario aprovechar este recurso e integrarlo como herramienta educativa: usar los videojuegos como herramienta cognitiva es una realidad que no se debe desperdiciar, ya que tienen un aporte muy enriquecedor para la actividad formativa (Sánchez i Peris, 2012). La relación videojuego-educación ha sido estudiada por multitud de autores, como Gee (2004), quien define 36 principios o formas de aprender con los videojuegos. Con su uso en educación, el alumnado no solo experimentaría el simple hecho de jugar para alcanzar una finalidad educativa, sino que contribuiría a diferentes aspectos como la mejora de los reflejos, la psicomotricidad, la iniciativa, la autonomía, la alfabetización digital, el desarrollo de una estrategia de pensamiento superior y la resolución de problemas (Cuenca y Martín, 2010), así como el establecimiento de relaciones causales, la toma de decisiones, o el análisis de valores y contravalores puestos en práctica a partir de un aprendizaje estimulante y práctico basado en la perspectiva didáctica de la teoría de Dewey (Sánchez i Peris et al., 2012).

Sin embargo, el uso de los videojuegos como herramienta educativa no está exento de críticas al considerarse fuente de sexismo o violencia. Otros autores, como Grossman (2000) y Provenzo (2001), ponen en duda su uso en el ámbito educativo, ya que creen que se impone como entretenimiento. No obstante, no todas las tendencias van en la misma sintonía, pues el Parlamento Europeo habla de los videojuegos como facilitadores del aprendizaje y como medios eficaces en algunas terapias (Extberria, 2012). Por otra parte, la investigadora Gros (2012) habla en su investigación de su integración en el sistema educativo formal. Por su parte, Esnaola y Levis (2008) apuntan que las investigaciones que dudan del uso de videojuegos como instrumentos educativos terminan postulando argumentos funcionalistas que no tienen en cuenta el valor de las narrativas audiovisuales como ejes del aprendizaje educativo, ya que estos estudios obvian que, cuando se 
utilizan dentro del aula, pueden constituir un aliado de primer orden como herramienta educativa (Pallares Piquer, 2013).

Por tanto, muchos autores señalan la necesidad de integrar el videojuego comercial en el aula, aunque muchos de ellos sean puramente instruccionales y no busquen con mayor insistencia el entretenimiento, lo que beneficiaría considerablemente el trabajo en el aula. En lo referente a los videojuegos de tipo histórico, y tras estudios particulares sobre ellos, se puede considerar que algunos de estos suponen memoria y no historia, debido a que no cuentan con un ideal de verdad y no están movidos por un interés de curiosidad. Además, tampoco pueden considerarse historia porque no acuden a fuentes primarias ni están insertos en un contexto general (Venegas, 2020). No obstante, el profesorado ha de mediar para que los estudiantes sean capaces de utilizar las características del videojuego de forma educativa, aprovechando enseñanzas que ya se han desarrollado de manera informal, para que trabajen a través del aprendizaje colaborativo y diseñen experiencias que vayan mucho más allá de lo conceptual. Es decir, es necesario superar su uso como un elemento puramente transmisor, puesto que la escuela no desempeña la función de antaño.Este ha de utilizarse como un material educativo que permite la introducción de contenidos a partir de un entorno de aprendizaje complejo, multidimensional, multimedia e interactivo (Pallares Piquer, 2013). Así, el videojuego en el aula debe de estar orientado a conseguir objetivos que otras metodologías no consiguen (Mugueta et al., 2015) y el profesorado debería actuar como catalizador de medios, ayudando a construir redes de aprendizaje que colaboren en la construcción del conocimiento. Es por ello que se destaca la importancia de la formación del profesorado para que esto sea posible.

El uso de los videojuegos como herramienta educativa en el ámbito de la historia ha sido estudiado por diferentes investigadores en los últimos años. Destacan autores como MartínCáceres y Cuenca (2019) e Iturriaga (2015), así como las recientes investigaciones de Jiménez (2020) y Venegas (2020). Si bien muchos docentes son reacios al uso de videojuegos en el aula por considerarlos contraproducentes, es una realidad del siglo XXI y no podemos despreciar esta herramienta, sino que debemos seguir investigando para que se convierta en un recurso de la educación formal, ya que estos es un excelente medio de aprendizaje (Exteberria, 2008). Los videojuegos históricos suponen, en palabras de Venegas (2020), un recuerdo del pasado o memoria y no una historificación, sin negar que puedan considerarse historia. Habría que destacar que los estos videojuegos suponen una herramienta fundamental para entender el pasado $y$, por lo tanto, se comprende su uso como fuente histórica.

Como se ha afirmado anteriormente, el uso de los videojuegos ocupa un gran número de horas entre los adolescentes (AEVI, 2020). Entre ellos tienen mucho éxito los de carácter histórico, algo que plantea una contradicción con respecto a la visión de la asignatura en el contexto formal, por lo que este entusiasmo puede deberse al uso de la tecnología para este tipo de contexto. Son diferentes los soportes electrónicos que permiten acceder a los videojuegos, algo que contribuye a su mejor adaptación al aula. Sin embargo, estos no están considerados como educativos, ya que se contemplan desde una perspectiva lúdica $y$, como afirman Jiménez y Cuenca (2015), se necesita un gran conocimiento para poder ser usados en el aula, dado que suponen un alejamiento del sistema tradicional de enseñanza.

Los videojuegos de tipo histórico ayudan a que el alumnado se relacione activamente con el pasado de una forma en la que ningún otro medio puede ofrecer hasta la fecha (Venegas, 2019). 
Estos se centran normalmente en un periodo histórico concreto o en varios de ellos, produciéndose un proceso de evolución, lo que lo hace atractivo desde el punto de vista lúdico y educativo. Aunque su principal función es la diversión, el tratamiento con el que esté confeccionado puede proporcionar claves históricas en torno a las diferentes etapas de la historia y su evolución, siempre teniendo en cuenta la ambientación, las incongruencias o los anacronismos que puedan confluir dentro del juego en cuestión.

Esta tipología de videojuegos no solo permite la inmersión en la historia que se narra virtualmente, sino que aumenta la concentración hacia lo que el alumnado está haciendo a través de la participación activa (Eguia et al., 2013), convirtiéndolos en investigadores que experimentan con el mismo en las sociedades como si de un laboratorio socio-histórico se tratase. Aspectos como la ambientación realista contextual a nivel de paisajes y entornos fielmente representados en los videojuegos, y los estudios en relación a momentos y hechos históricos para caracterizar fidedignamente personajes, eventos y escenarios, son trabajados en el videojuego a través de principios de temporalidad, espacialidad, conflicto-consenso, cambio-continuidad, intencionalidad-racionalidad, interdependencia, causalidad e identidad (Muñoz, 2012). Esto permite su uso para trabajar la multicausalidad de la historia, sobre todo en aquellos que gestionan sociedades y civilizaciones (Mugueta et al., 2015).

Como afirma lturriaga (2015), otro punto a su favor es que la experiencia a la hora de jugar es intensa, como no lo es cualquier otro recurso que podamos utilizar para la enseñanza de la historia. Esto es debido a que la recreación de las atmósferas de los edificios, los objetos, los sonidos, etc. es simplemente impecable, y ayuda a que al alumnado se sienta protagonista de la historia en la cual está jugando y aprendiendo al mismo tiempo. Así, la ubicación espaciotemporal es fundamental para dotar del conocimiento que se pretende adquirir, pudiendo estar acompañado de representaciones geográficas y mapas de alta calidad que enmarcan dicho contexto, características del entorno físico y elementos claves del paisaje, así como elementos urbanos y signos sociales que logran reproducir fielmente paisajes de la historia. Todo esto hace de los videojuegos históricos una herramienta para la enseñanza y aprendizaje de la historia (Gálvez, 2006).

Las nuevas tecnologías en general, y los videojuegos en particular, se prestan perfectamente al desarrollo de algunas habilidades del pensamiento histórico, puesto que son herramientas que se centran en un momento o periodo histórico determinado, donde se desarrollan simulaciones de los procesos de evolución histórica que los hace especialmente útiles y atractivos (lturriaga, 2015).

\section{Metodología}

Este trabajo se ha basado en la experimentación a través del uso del videojuego de carácter lúdico Dominations, utilizando el mismo como herramienta didáctica para trabajar el sentido social de la historia, los procesos de evolución histórica, la distinción de periodos, la experimentación de los cambios que se producen en las sociedades, y el conocimiento y la valoración del patrimonio y el medioambiente. La experimentación partió del recurso principal del uso del videojuego, aunque en el proceso de aprendizaje se ha combinado el uso de este con otras fuentes de información, como el libro de texto e Internet. Dentro del ámbito de la didáctica de la historia, esta tipología de videojuego aporta conceptos de evolución histórica, es decir, sobre los 
cambios y las permanencias que se producen en la sociedad, sobre la relación de esta con el medio en lo referente a las formas de contacto intercultural y el aprovechamiento de los recursos naturales. Es posible tomarlo como punto de partida para el desarrollo de cualquier sociedad y como elemento básico de conflictos entre civilizaciones. En este tipo de videojuegos, se parte del periodo prehistórico, pudiéndose avanzar hasta la actualidad e incluso hasta periodos futuros, destacándose su implicación de aspectos socioculturales básicos como la evolución social y tecnológica, la diversidad de las civilizaciones en función a las diferentes características culturales, los cambios y permanencias, y las relaciones comerciales y políticas (Cuenca, 2012).

\subsection{Objetivos}

Esta investigación tuvo una serie de objetivos que pretendieron comprobar las ventajas que posee el empleo del videojuego Dominations por parte del alumnado para aprender contenidos específicos acerca de la civilización mesopotámica (aspectos socioeconómicos, culturales, políticos, patrimoniales, etc.) y los beneficios de su uso en el aula para la enseñanza de la misma, resaltando las habilidades positivas que el alumnado ejercita para lograrlos. Se pretendió, pues, investigar si el alumnado consigue aprendizajes significativos con el uso del videojuego Dominations, desplegándose de este diferentes objetivos específicos en torno a los conceptos clave a ser analizados (Tabla 1).

\section{Tabla 1}

\section{Conceptos clave y objetivos específicos de la investigación}

Concepto clave Objetivos específicos

1. Sociedad y economía

2. Tiempo histórico

3. Contexto geográfico

4. Uso como fuente

5. Patrimonio
1.1. Analizar si el estudiante es capaz de observar las características socio-histórica del período mesopotámico, así como el establecimiento y difusión de la cultura urbana usando el videojuego como laboratorio de datos

1.2. Evaluar la capacidad del estudiante de investigar la organización social, política y económica a partir del mantenimiento de la sociedad en el videojuego

2.1. Analizar cómo el estudiante experimenta el proceso de evolución histórica a través del uso del videojuego

2.2. Valorar si el estudiante es capaz de distinguir las etapas históricas (Prehistoria-Edad Antigua) a través de la evolución y la progresión en el uso del videojuego

3. Analizar si el estudiante consigue conocer el medio físico y urbano a través de los mapas del videojuego

4. Valorar el uso del videojuego como fuente de información para reconocer el sentido social de la Historia y valorar la conservación, el patrimonio y el medio ambiente

5.1. Comprobar si el estudiante es capaz de apreciar los diferentes elementos patrimoniales a través del uso del videojuego 
5.2. Analizar si el estudiante valora el patrimonio en el desarrollo de la sociedad a través de las diferentes manifestaciones culturales urbanísticas

Fuente: Elaboración propia.

\subsection{Diseño de la investigación}

Para abordar los objetivos señalados, se consideró que la metodología más apropiada para investigar el alcance educativo del videojuego en este contexto se enmarca en el paradigma interpretativo, dentro de perspectivas como la etnológica y la concepción holística que propone el enfoque cualitativo (Herrán, 2005). Se emplearon técnicas de carácter cualitativo, debido a que este enfoque intenta comprender la conducta en las personas que participan y se interpretan los significados que resultan de su interacción con un objeto (en esta investigación, el videojuego) para realizar una descripción de las consecuencias de dicha interrelación (Martínez Rodríguez, 2011 ). Asimismo, aporta una copiosa información descriptiva de actividades, procesos, efectos y participantes implicados en un acto ámbito social (Del Rincón et al., 1995). Todo ello se realiza a través del análisis de una experiencia educativa evaluada a partir de diversos instrumentos, que serán detallados en los siguientes apartados.

\subsection{Contexto de la investigación}

El contexto elegido fue el primer ciclo de la Educación Secundaria Obligatoria, concretamente el primer nivel ( $1 .^{\circ}$ de ESO), ya que es durante este curso en el que se trabaja la Edad Antigua dentro de la asignatura Geografía e Historia, concretamente el contenido relacionado con la cultura mesopotámica. Para la investigación ha participado una clase completa, con un total de 25 estudiantes: 14 alumnos y 16 alumnas en edades comprendidas entre los 12 y los 14 años. Dentro de la heterogeneidad de la clase, cabe resaltar a cuatro menores que no promocionaron de curso, dos jóvenes con trastorno por déficit de atención e hiperactividad (TDAH), un menor con dislexia, dos estudiantes con adaptaciones curriculares no significativas y un joven con adaptación curricular significativa. Esta diversidad en el conjunto de la clase, que, a priori, podría implicar dificultades a la hora de llevar a cabo las actividades o de la adquisición de los contenidos tratados, resultó, como se podrá apreciar en el apartado de discusión, ser beneficiosa para el conjunto de los estudiantes, pues aumentó la reciprocidad y la cooperación entre ellos.

\subsection{Técnicas de recogida de la información}

Para el desarrollo del estudio, se elaboraron dos técnicas de recogida de la información: una tabla de observación, que recoge diversas categorías con la que se pretende medir el grado de aprendizaje de los contenidos propuestos a través del videojuego, y un cuestionario diseñado ad hoc. La tabla de observación fue utilizada para analizar la experiencia educativa durante su implantación en el aula a través de la observación participante y, tras la misma, a través de la visualización y análisis de las grabaciones en vídeo. Dicha tabla de observación gira en torno a cinco conceptos clave o categorías (Tabla 2), siendo la primera de ellas la enfocada a los contenidos relacionados con la sociedad y la economía de la cultura mesopotámica, la segunda relacionada con el tiempo histórico, la tercera con el contexto geográfico, la cuarta asociada al uso del videojuego como herramienta de aprendizaje, y la quinta con cuestiones patrimoniales relacionadas con la valoración y la conservación del patrimonio. En esta, además, se anotaron los 
datos referentes al comportamiento en el aula, añadiéndose un espacio reservado para las anotaciones de los descriptores necesarios y para los comentarios que estimara oportuno el profesorado. Con el uso de este instrumento, se pretendió analizar el impacto educativo del videojuego para conocer el grado de aprendizaje de los contenidos específicos de la unidad de la cultura mesopotámica a través de la observación y la producción del alumnado.

\section{Tabla 2}

Categorías o conceptos clave, objetivos específicos a los que dan respuesta (OE), indicadores de logro y descriptores definidos

Categorías OE Indicadores Descriptores

\begin{tabular}{llll}
\hline $\begin{array}{l}\text { 1. Sociedad y } \\
\text { economía }\end{array}$ & 1.1 & $\begin{array}{l}\text { No las } \\
\text { observan }\end{array}$ & $\begin{array}{l}\text { El alumnado no observa las características socio- } \\
\text { históricas -nomadismo, sedentarización, caza, recolección, } \\
\text { división de tareas, agricultura y jerarquía- del período } \\
\text { mesopotámico. }\end{array}$
\end{tabular}

Las observan El alumnado observa algunas características socioparcialmente históricas como la caza y la sedentarización entre otras, sin profundizar en el establecimiento de la cultura urbana.

Las observan El alumnado observa las principales características sociototalmente históricas del período mesopotámico así como lo referente a la difusión de la cultura urbana.

1.2 Elemento El alumnado solo usa el videojuego como elemento de lúdico diversión y no de investigación.
Elemento El alumnado usa el videojuego como un incentivo para motivacional investigar, pero sin aprovechar al máximo sus posibilidades.

Elemento El alumnado usa el videojuego para investigar la investigador organización socio-política y económica, concediéndole gran importancia en el desarrollo de la sociedad.

$\begin{array}{lll}\text { 2. Tiempo } & 2.1 & \text { No lo } \\ \text { Histórico } & \text { experimenta }\end{array}$

El alumnado no experimenta el proceso de evolución histórica obviando las etapas por las que pasan en el videojuego -Edad Primitiva, Edad del Hierro y Edad del Bronce- y su relación con etapas históricas -Paleolítico o Neolítico- así como los cambios principales que se producen en cada etapa -nuevas construcciones o inicio de la agricultura-.

Lo experimenta El alumnado experimenta algunos procesos de la parcialmente evolución histórica reconociendo algunas por las que pasan en el videojuego -Edad Primitiva o Edad del Hierro- y su relación con etapas históricas identificando algunos cambios que se producen en cada etapa. 
Lo

experimenta

totalmente

2.2 Ninguna

distinción

Alguna

distinción

Total

distinción

3. Contexto

Geográfico

4. Uso del videojuego

5. Patrimonio

5.1 Etnológico

Natural

Histórico

Artístico

Holístico

Lo conoce
El alumnado experimenta todos los procesos de la evolución histórica reconociendo las diferentes etapas y relacionándolas con la historia e identificando multitud de cambios que se producen en cada una de las mismas.

El alumnado no hace distinción entre periodos históricos Prehistoria y Edad Antigua- así como etapas históricas ya trabajadas con anterioridad -Paleolítico, Neolítico-.

El alumnado hace distinción entre periodos históricos Prehistoria y Edad Antigua- así como cita alguna etapa de la Prehistoria, pero sin ofrecer muchos detalles de los períodos ni las etapas.

El alumnado hace distinción entre los periodos históricos y las etapas con mucho detalle.

El alumnado desconoce el contexto mesopotámico

El alumnado conoce parcialmente el contexto de la civilzación mesopotámica (localización en el mapa, principales ríos que rodean a la cultura).

El alumnado conoce con profundidad el contexto de la civilización mesopotámica aportando localizaciones (Ur, Uruk, Babilonia) y datos concretos sobre el medio físico (Creciente Fértil) y sus principales ríos-.

El alumnado no emplea el videojuego como fuente de información, sino de forma lúdica.

Lo emplea El alumnado emplea parcialmente el videojuego como parcialmente fuente de información, pero se distrae por su componente lúdico.

Lo emplea El alumnado emplea total el videojuego como fuente de totalmente información.

Apreciación de elementos festivos, tradicionales y con paisajes asociados.

Apreciación con elementos de carácter medioambiental.

Apreciación con elementos documentales y arqueológicos.

Apreciación de manifestaciones correspondientes a diferentes movimientos artísticos.

Apreciación indiferenciadas con diversas manifestaciones patrimoniales. 
5.2 No lo valora El alumnado no valora el patrimonio en el desarrollo de la sociedad.

Lo valora El alumnado valora algunos elementos del patrimonio en parcialmente el desarrollo de la sociedad.

Lo valora El alumnado valora totalmente el patrimonio en el totalmente desarrollo de la sociedad.

Nota: Los objetivos específicos (OE) aparecen definidos en la tabla 1 (vid. Supra).

Fuente: Elaboración propia.

El segundo instrumento diseñado consistía en un cuestionario que incluyó 22 preguntas de tipo abierto (Tabla 3). Estas se contestaban tras el uso del videojuego y pretendía evaluar el impacto educativo obtenido en relación a los contenidos clave o categorías seleccionadas: distinguir las etapas históricas (Prehistoria-Edad Antigua) aparecidas, reconocer las características sociohistóricas del período mesopotámico, conocer el establecimiento y difusión de su cultura urbana usando el videojuego como laboratorio de datos, experimentar el proceso de evolución histórica, emplear el videojuego como fuente de información para reconocer el sentido social de la Historia e investigar las organizaciones social, política y económica que mantenían la sociedad, así como resaltar la importancia del patrimonio y reconocer las diferentes manifestaciones culturales y artísticas. Así, el cuestionario abarcaba contenidos exclusivos que aparecían durante el propio uso del videojuego y otros que, si bien estaban relacionados con él, necesitaban de otras fuentes de información para su resolución.

Tal instrumento se encuadró dentro de las herramientas cualitativas, concretamente del tipo investigación-acción, debido a que es una modalidad de investigación que propone, en el caso de la educación, una forma de estudiar y explorar una situación educativa con la finalidad de mejorarla. Este tipo de investigación no solo se presenta como un método de investigación, sino como una herramienta encaminada hacia el cambio educativo, en donde el docente investigador es sujeto activo de su propia practica (Cañal et al., 2005), y, además, aporta valiosos datos descriptivos de los ámbitos o contextos educativos, actividades y creencias de los participantes en los escenarios educativos (Goetz y Lecompte, 1988).

\section{Tabla 3}

\section{Cuestionario para evaluar los contenidos de la unidad introducidos a través del} videojuego

\section{Enunciado}

1. ¿̇En qué edad comienza vuestra partida?

2. ¿Podríais decirme los elementos naturales que están alrededor de vuestro poblado?

3. ¿Es vuestra sociedad nómada o sedentaria? Razonad la respuesta.

4. Describid vuestro asentamiento, diciendo qué edificios tiene y para qué sirven cada uno

5. ¿Creéis que todos los edificios que aparecen en el videojuego corresponden a esta edad? ¿Por qué? 
6. ¿Cómo se alimenta vuestra sociedad? Enumerad los productos que sirven de alimento para tu sociedad e investigad si en la sociedad mesopotámica se injerían estos alimentos.

7. ¿Qué impacto tiene para la naturaleza el hecho de ampliar vuestro pueblo? ¿Creéis que la naturaleza era importante para los habitantes de Mesopotamia? ¿Creéis que la naturaleza es importante ahora para nosotros y nosotras?

8. ¿̇Habéis construido algún edificio más? ¿De qué tipo es? ¿Para qué sirve?

9. ¿̇s han atacado? ¿Qué edificios usaréis para defenderos?

10. Pasad de edad. ¿Cómo se llaman los diferentes períodos que aparecen en el videojuego? Ordenadlos en un eje cronológico.

11. ¿Qué cambios observáis que ha sufrido vuestro pueblo al avanzar de edad?

12. ¿Qué características son relevantes en cada nueva edad? Haced un esquema.

13. ¿Qué nuevos edificios encontráis al avanzar de edad?

14. Para conseguir más recursos habréis tenido que luchar con otras tribus. Anotad los nombres de los lugares donde habéis luchado con vuestro ejército y buscad información sobre ellos.

15. ¿Crees que la religión está presente en el videojuego? Buscad los elementos característicos si fuera necesario en vuestro libro.

16. ¿Cuál es la base de vuestra economía? ¿Corresponde con la base de la economía de Mesopotamia?

17. ¿En qué periodo podéis comenzar las labores de agricultura?

18. ¿Creéis que existen similitudes entre los alimentos de vuestro pueblo y los que encontramos actualmente?

19. Investigad sobre la Maravilla que tenéis que construir y explicad qué función tenía dentro de la sociedad.

20. Describid vuestra sociedad. ¿̇Todos hacen las mismas funciones? ¿Quién dirige tu sociedad? ¿Creéis que la sociedad está completa? Completad la información si fuera necesario con vuestro libro de texto.

21. ¿2Reconocéis los elementos patrimoniales de vuestro poblado? Enumeradlos y decid por qué creéis que son patrimonio.

Fuente: elaboración propia.

\subsection{Videojuego y secuencia formativa}

El juego Dominations es una aplicación de software libre y descarga gratuita lanzado el 1 de abril de 2015 para las plataformas de Android, Windows e iOS, creado por la plataforma NEXON, una compañía de Corea del Sur que desarrolla y distribuye videojuegos en línea, fundada en Seúl en 1994, aunque con sede localizada en Tokio (Japón). Este videojuego está 
basado en la estrategia y se encuadra dentro de los juegos donde la Historia ocupa el eje central con un desarrollo que atiende a una gran diversidad de aspectos, proporcionando una visión integral de la sociedad y atendiendo aspectos de evolución, pues consta de nueve edades: desde la edad primitiva hasta la edad industrial. Los contenidos que se trabajan en el juego son de gran diversidad, destacando los de carácter históricos y geográficos, puesto que aparecen reflejados lugares históricos como Ur, Babilonia o el río Nilo, los contenidos urbanísticos y patrimoniales-artísticos, pues aparecen referentes histórico patrimoniales como Stonehenge o los Jardines Colgantes de Babilonia, y los contenidos sociales y económicos, como el paso de la economía cazadora-recolectora a una economía productora. Estos proporcionan una visión integral de las sociedades, fundamentan el marco histórico y las claves para identificar y diferencias diferentes periodos históricos y permite situarse en un espacio y tiempo determinado e incluyen el proceso de evolución histórica de las diferentes culturas o civilizaciones, siendo muy completo desde el punto de visto educativo (Cuenca, 2012).

La programación de la unidad se configuro teniendo como eje principal el videojuego señalado, Dominations. A este le acompañaron cuatro actividades donde se intercalaron el uso del videojuego y el libro de texto de Geografía e Historia (Ayuela, 2016) con diversas estrategias metodológicas como la investigación o el trabajo cooperativo (Tabla 4).

Tabla 4

Secuenciación de las actividades llevadas a cabo en el aula y objetivos específicos $(\mathrm{OE})$ asociados

\begin{tabular}{|c|c|c|c|}
\hline Nombre & N. ${ }^{\circ}$ Sesiones & Desarrollo & OE \\
\hline $\begin{array}{l}\text { ¿Qué sabemos } \\
\text { de } \\
\text { Mesopotamia? }\end{array}$ & 1 & $\begin{array}{l}\text { La actividad está dividida en dos partes: la primera } \\
\text { un cuestionario inicial de conocimientos previos, } \\
\text { mientras que la segunda es una presentación de las } \\
\text { caracteristicas generales de la unidad y las } \\
\text { herramientas que se utilizaran. }\end{array}$ & \\
\hline $\begin{array}{l}\text { Investigando } \\
\text { nuestra sociedad }\end{array}$ & 3 & $\begin{array}{l}\text { De forma grupal, el alumnado experimenta la } \\
\text { evolución de los periodos historicos a traves del uso } \\
\text { del videojuego como un laboratorio de simulación } \\
\text { socio-histórico. Para evitar que los objetivos de la } \\
\text { unidad se pierdan, se planteará al alumnado una } \\
\text { serie de cuestiones que estarán relacionadas con la } \\
\text { misma; para ello, deberán recoger datos, buscar } \\
\text { información y tomar capturas de pantalla que sirvan } \\
\text { de ejemplo. Estos, juntos con otros datos recogidos, } \\
\text { formarán parte de la siguiente actividad que } \\
\text { tendrán que elaborar. }\end{array}$ & $\begin{array}{l}1.1 \\
1.2 \\
4\end{array}$ \\
\hline $\begin{array}{l}\text { La carpeta } \\
\text { cuneiforme }\end{array}$ & 3 & $\begin{array}{l}\text { La tercera actividad se elabora una carpeta que } \\
\text { contendrá diferentes tareas basadas en la } \\
\text { información que previamente el alumnado haya } \\
\text { recopilado en la fase de búsqueda de información a } \\
\text { través de la experimentación con el videojuego. Las } \\
\text { tareas son un eje cronológico (donde detallen todos } \\
\text { los periodos que han experimentado a través del } \\
\text { videojuego), las preguntas que anteriormente se les }\end{array}$ & $\begin{array}{l}2.1 \\
2.2 \\
3 \\
5.1 \\
5.2\end{array}$ \\
\hline
\end{tabular}


había planteado, y la investigación sobre los lugares y el patrimonio que han conocido a traves del videojuego.

¿Qué hemos 3 aprendido?
Por último, y como actividad de sintesis, el alumnado prepara una exposición oral en grupo a partir de la información obtenida. En la exposición tienen que detectar incongruencias y anacronismos entre lo que han experimentado a través del videojuego y los contenidos acerca de la civilización mesopotámica del libro de texto.

Fuente: elaboración propia.

\section{Resultados}

Los resultados obtenidos tras el desarrollo de la investigación descrita se presentan a continuación conforme a los contenidos clave y objetivos propuestos, siendo estos los que estructuran este apartado. Los datos específicos se expresan numéricamente y se especifica en cada uno de los objetivos qué porcentaje del grupo de estudio alcanza cada indicador. Se acompañan estas cuantificaciones con una explicación concisa que describe con exactitud la realidad experimentada.

En primer lugar y en relación al concepto clave 1, Sociedad y economía, se pretendía observar si el alumnado reconocía las características socio-históricas del periodo mesopotámico, así como el establecimiento y difusión de la cultura urbana usando el videojuego como laboratorio de datos (OE 1.1.).

Por ello, en lo relativo a este objetivo, un total de 17 alumnos (68\%) observaron algunas características de la cultura mesopotámica de forma parcial. Por ejemplo, señalaron las incongruencias en las estructuras urbanísticas (las casas o el centro urbano), pues no se correspondían al período que estaban experimentando. Igualmente, hicieron referencia a la sociedad desde el punto de vista de la división de tareas, explicando detalladamente las mismas y especificando tales como la recolección, la caza, la construcción y la guerra. Por otro lado, un total de 8 alumnos (32\%) realizaron una observación más profunda relacionando los primeros asentamientos al Neolítico y la creación de primeras ciudades con un periodo posterior, así como fueron capaces de establecer una relación entre la Edad de los Metales y el inicio de las ciudades.

En general, la totalidad del alumnado no tuvo problemas a la hora de explicar la jerarquía social, ya que se autoidentificaron como gobernantes o personas de poder en la pirámide social, al controlar en gran medida las decisiones de su sociedad. Por otro lado, ningún estudiante dió detalles sobre la religión, aunque sí proporcionaron explicaciones sobre su existencia al detectar templos y esculturas. En otras cuestiones, hicieron referencia a la sedentarización como modo de vida y a la agricultura como base de la economía. Cabe destacar a 6 alumnos (24\%) que observaron que había transacciones comerciales con oro, pero lo detectaron como error histórico tras comprobar en el libro de texto que estas se hacían realmente con piezas de plata. 
Relacionado con este primer concepto, se analizaba si el alumnado era capaz de investigar la organización social, política y económica a partir del mantenimiento de la sociedad en el videojuego (OE 1.2.). En lo concerniente a este objetivo, 17 alumnos (68\%) hicieron un uso motivacional del videojuego, es decir, lo usaron para investigar las cuestiones planteadas a partir del mantenimiento de la sociedad, pero no lo aprovecharon totalmente como recurso para alcanzar un nivel de información mayor. Este grupo detectó los principales elementos socioeconómicos, describiendo la sociedad como sedentaria y asociando este concepto con el crecimiento de las construcciones (las casas, el centro urbano) y la agricultura, a la que reconocen como principal base económica. A esta actividad le añadieron otras actividades como la caza, el comercio con caravanas (incongruencia), las minas y el saqueo de otras aldeas. El resto del grupo, sin embargo, hizo un uso más profundo del videojuego, describiendo, además de todo lo anterior, el comercio como actividad económica.

Un segundo concepto clave a analizar era el tiempo histórico. En concreto, el OE2.1. buscaba analizar la capacidad del estudiante de observar el proceso de evolución histórica a través del uso del videojuego. Para este objetivo, el alumnado experimentó en su totalidad tres edades propuestas en el videojuego -Edad Primitiva, Edad de Hierro y Edad de Bronce-.

Un $52 \%$ del grupo $(n=13)$ experimentó la evolución histórica de manera más parcial y profundizando menos en las características: señalaron datos como el nombre de las etapas, la cronología o la evolución urbana. Por otro lado, un $48 \%$ del alumnado, además de aportar lo mencionado anteriormente, profundizó en otros aspectos como el urbanismo, la economía (la agricultura), o el tipo de sociedad (nómada o sedentaria). Como consecuencia de la práctica para alcanzar este objetivo, se realizó un eje cronológico ilustrado con capturas de pantalla del videojuego en las que se ordenaron las etapas. A través de esta actividad, el alumnado explicó brevemente que los cambios antes señalados se producían al evolucionar de un periodo a otro, coincidiendo la totalidad del ellos en las mejoras de las construcciones y nuevos edificios, así como la inclusión de la agricultura como algo importante que marca la evolución histórica.

De la misma manera, se pretendía conocer si el alumnado era capaz de distinguir las etapas históricas (Prehistoria- Edad Antigua) a través de la evolución y la progresión en el videojuego (OE 2.2.). Con respecto a este objetivo, la totalidad del alumnado respondió correctamente a las cuestiones propuestas a resolver con el videojuego, pero en ninguno de los casos distinguieron con claridad las etapas históricas (Prehistoria-Edad Antigua), ni aportaron datos concretos de los contenidos históricos trabajados con anterioridad. En todas las respuestas, para hacer referencia a distinciones entre las etapas históricas, se hicieron alusiones nuevamente a la modificación de estructuras relacionadas con la ciudad para responder al principio de evolución histórica. Es decir, en su mayoría relacionaron la evolución con el cambio y/o modernización de los edificios y no con otros aspectos como el avance tecnológico.

Un tercer elemento clave se centraba en el análisis del contexto geográfico: sobre esta temática, se buscaba evaluar si a través de los mapas del videojuego el alumnado era capaz de conocer el medio físico y urbano de Mesopotamia (OE 3). Para este objetivo, el alumnado tuvo la oportunidad de conocer el contexto geográfico mediante la interacción con el videojuego a partir de localizaciones geográficas donde se asentaba la cultura mesopotámica (como, por ejemplo, Ur, el río Éufrates, Babilonia o el Creciente Fértil) y a partir de los enfrentamientos con otros pueblos. Así, la cartografía incluida en el videojuego permitió la profundización geográfica de 
la cultura mesopotámica gracias a la exploración de diferentes enclaves históricos, así como su relación con los países que reemplazan en la actualidad. Un total de 19 estudiantes (76\%) fueron capaces de transmitir con un alto grado de detalle el contexto geográfico, lo que permitió la indagación de lugares históricos variados haciendo uso de otras fuentes de información. Sin embargo, un grupo de 6 alumnos $(24 \%)$ se limitó a la información que proporcionaba el videojuego, siendo esta conceptual o escasa para este objetivo.

El cuarto concepto perseguía analizar el uso del videojuego como fuente de información para el estudio de los contenidos de la unidad (OE 4). El alumnado casi en su totalidad hizo uso del videojuego como herramienta principal de la unidad: 8 alumnos (32\%) hicieron un empleo riguroso del mismo a la hora de la búsqueda de información, mientras que un total de 17 estudiantes $(68 \%)$ tuvieron una actitud más laxa en su análisis. Los estudiantes que realizaron un uso adecuado del videojuego como fuente $(n=8)$ resolvieron satisfactoriamente las tareas propuestas sabiendo combinar este con otras fuentes como el libro de texto o internet (en aquellos casos necesarios).

El quinto y último contenido se centraba en el análisis del elemento patrimonial, concretado por el OE 5.1., centrado en valorar si el estudiante era capaz de apreciar los diferentes elementos patrimoniales a través del uso del videojuego. Este objetivo ha sido el que más problemática ha presentado, puesto que 8 estudiantes $(32 \%)$ no aportaron datos suficientes para su valoración debido a que no estaba claro el concepto de patrimonio, pese haber sido trabajado en clase con anterioridad. Asimismo, un total de 17 estudiantes (68\%) distinguieron como patrimonio uno de los edificios a construir en el desarrollo de la partida en el videojuego como era los Jardines Colgantes de Babilonia, por lo que relacionaron el patrimonio con su vertiente artística en base a la monumentalidad, la arquitectura y belleza que representa. De esos 17 estudiantes, 11 de ellos lo apreciaron como patrimonio artístico, mientras que otros 6 estudiantes lo clasificaron como patrimonio artístico y natural tras haber investigado en profundidad. Por tanto, se pudo concluir que tanto el término patrimonio en sí, previamente trabajado en el aula, como la clasificación de los diferentes tipos de patrimonio supone una complejidad en este nivel, además de que su apreciación en el videojuego se resume a lo puramente material.

Junto al anterior, se buscó evaluar si el alumnado era capaz de valorar el patrimonio en el desarrollo de la sociedad a través de las diferentes manifestaciones culturales urbanísticas (OE 5.2). En base a lo tratado en el objetivo anterior, 19 estudiantes $(76 \%)$ hicieron una valoración parcial al centrarse solo en los Jardines Colgantes de Babilonia como único elemento detectado del patrimonio. Este elemento patrimonial ha sido investigado por el alumnado, señalando como elementos importantes en tal conjunto la naturaleza y el medio físico, por lo que indirectamente hicieron referencias al patrimonio natural $y$, por ende, lo apreciaron como patrimonio dentro de un conjunto artístico mayor, aunque sin relacionarlo con el patrimonio en sí. Sin embargo, los 6 estudiantes restantes (24\%) no aportaron datos para valorar el patrimonio.

\section{Reflexiones finales}

En la práctica de esta investigación, el uso del videojuego Dominations ha demostrado ser una herramienta excelente para distinguir edades históricas. El alumnado ha podido experimentar desde la prehistoria hasta la cultura mesopotámica y analizar los cambios y permanencias que se van produciendo en su desarrollo. El alumnado ha podido interactuar con entornos históricos 
así como ensayar con la historia como si de un laboratorio se tratase (Cuenca et al, 201 1). Aún así, no se ha logrado una mayor concreción dentro de las etapas, dado que el alumnado relaciona la evolución con la modernización de las estructuras urbanísticas y no con otros aspectos. Por tanto, el videojuego proporciona información sobre los avances generales en cada período histórico, pero sin profundizar en otros aspectos.

En lo referente al contenido socio-histórico, se corresponde en gran medida con los conocimientos previos que el alumnado debe de poseer sobre la Prehistoria, puesto que, a pesar de que detectan incoherencias a nivel urbanístico, reconocen a través del videojuego aspectos relevantes para el conocimiento de las culturas prehistóricas y antiguas, por lo que es un apoyo y potenciador del contenido para el profesorado cuando el alumnado tenga un aprendizaje significativo de cara a trabajar la evolución histórica (Jiménez-Palacios, 2020). Además, supone un recurso muy valioso para la motivación de del alumnado. Este es uno de los aspectos más destacables del videojuego en este tipo de contexto debido a que favorece el entusiasmo por las temáticas que se introducen (Arias et al., 2018) y supone la mejora la estimulación en el aula puesto que altera las emociones positivamente. El clima y el rendimiento de la clase se ve favorecido, obteniendo así mejores resultados dado que ayuda a tener menos conductas disruptivas y más agradables en el aula. Esto origina más concentración y entusiasmo y, por lo tanto, un aprendizaje más significativo (Jiménez-Palacios, 2020).

El videojuego supone también una herramienta capaz de detectar los principales elementos en relación al medio físico y que ayudan a contextualizar las más importantes civilizaciones antiguas en general y, particularmente, las hidráulicas. A la vez, proporciona nuevas informaciones sobre los principales núcleos mesopotámicos así como sobre la localización de ríos y valles que sirvieron para la vida de esta cultura. Asimismo, gracias a la cantidad de información que ofrece, favorece que el alumnado investigue y complete sus conocimientos a partir del propio videojuego. Ha permitido igualmente a los discentes adquirir conocimientos sobre la organización social y económica de la cultura mesopotámica a través de la detección de incongruencias entre la información obtenida en el videojuego y la que han obtenido a través de otros medios, como el libro de texto o Internet. A pesar de que el videojuego aporta información sobre elementos patrimoniales, el alumnado lo ha detectado en escasas ocasiones. Solo ha asociado la idea de patrimonio en asociación a la monumentalidad. No obstante, el videojuego supone un acercamiento a los principales referentes patrimoniales (Cuenca, 2012), y favorece la comprensión de la cultura tangible (Egea-Vivancos y Arias-Ferrer, 2020) teniendo como ejemplo el hecho de que uno de los edificios que aparece en el juego sea los Jardines Colgantes de Babilonia y que ha permitido al alumnado categorizar el patrimonio a través de la simbiosis de arquitectura y naturaleza.

Por tanto, después de la realización y evaluación de esta propuesta, se subraya la idoneidad de este tipo de recursos -los videojuegos- para la enseñanza de la historia, dado que se adapta a la modernidad que se requiere para el trabajo con grupos de estas edades a la vez de ser una herramienta que motiva al alumnado, que favorece el aprendizaje y que hace partícipe al estudiante del proceso de enseñanza, logrando así un aprendizaje significativo y funcional. También hay que destacar la importancia de este tipo de recursos que mezclan lo lúdico y didáctico y que ayuda al tratamiento de la diversidad e inclusión del alumnado con necesidades específicas de apoyo educativo, puesto que el grupo por completo, independientemente de sus capacidades, sabe desenvolverse con el videojuego. Se convierte pues en una herramienta 
inclusiva y de apoyo. En lo concerniente a la consecución de los objetivos marcados, se alcanzaron con plenitud, pues el alumnado no solo ha experimentado con el videojuego, sino que ha alcanzado grandes niveles de conocimientos al compatibilizar la información con otros soportes, algo que quedó recogido en la evaluación final de la unidad. Sin embargo, el uso del videojuego ha traído una serie de limitaciones durante la puesta en práctica debido a la falta de apoyos tecnológicos de las familias con menos recursos económicos, lo que provocó que se tuvieran que reducir el número de grupos, siendo estos más numerosos.

Con respecto a la atención a la diversidad del aula, resulta interesante como el uso del videojuego con el alumnado que presentaba dificultades de aprendizaje ha permitido su acercamiento al grupo a través de la interacción, proponiendo nuevas formas de relación social, potenciando la comunicación donde todas las personas participantes aporten saberes y compartan experiencias; así, el videojuego ha funcionado como facilitador de intercambios y generador de espacios de reflexión entre el grupo estudiantil en torno al mismo, transformando el contexto en un entorno inclusivo que funcionan como una comunidad de práctica, además de ser usado para practicar habilidades o asistir el aprendizaje (Monjelat y Méndez, 2012).

En resumen, se puede afirmar que el videojuego Dominations es un recurso apto e idóneo para apoyar la labor del profesorado en las sesiones en el aula. Ahora bien, es importante recordar que dicho videojuego fue integrado en una secuencia formativa creada específicamente por el docente donde el videojuego era un recurso más pero no el único (Jiménez-Palacios, 2020). La correcta contextualización del juego en el proceso de enseñanza-aprendizaje favorece su aplicabilidad y la obtención de resultados favorables respecto a su aplicación (Egea-Vivancos y Arias-Ferrer, 2020).

Para concluir, y con respecto a las limitaciones de esta investigación, hay que resaltar que, por un lado, el videojuego es de tipo comercial y no se engloba en los denominados "serious games", por lo que el peso de la acción educativa para subsanar incongruencias históricas e incentivar la indagación es fundamental. Por otro lado, la muestra es reducida al tratarse únicamente de un grupo-clase donde el docente ejercía el rol de investigador. La ampliación de la muestra y la observación participante por parte de jueces externos a la investigación aportarían una mayor consistencia a los resultados. Aún así, esta investigación es una propuesta que abre posibilidades de futuras líneas, en especial, en el ámbito de la enseñanza de la historia a través de los videojuegos.

\section{Agradecimientos y financiación}

Está trabajo no ha obtenido financiación en ningún concurso público.

\section{Contribución específica de los autores}

El autor firmante del artículo es el único responsable del desarrollo de la investigación.

\section{Bibliografía}

AEVI (2020). La industria del videojuego en España. Anuario 2019. Recuperado de http://www.aevi.org.es 
Anderson C. A. Anderson, Sakamoto, A., Gentile, D. A., Ihori, N., Shibuya, A., Yukawa, S., Naito, M., Kobayashi, K. (2008). Longitudinal Effects of Violent Video Games on Aggression in Japan and the United States. PEDIATRICS. Oficial Journal of the American Academy of Pediatrics, 122(5), 1067-1072. https://doi.org/10.1542/peds.2008-1425

Arias Ferrer, L., Egea Vivancos, A. y García López, A. (2018). Aprender historia a través del juego de realidad virtual inmersiva "Carthago Nova". Propuesta de integración de un Serious Game en el proceso de enseñanza-aprendizaje. Clío. History and History teaching, 44, 26-40. http://clio.rediris.es/n44/articulos/monografico2018/03MonAriasEgeaGarcia.pdf

Ayuela, A. (2016). Geografía e Historia 1. ESO. Algaida

Barbier, B. (2014). Video Games and Heritage: Amateur Preservation?. Revue Des Arts et Médiations Humaines, 1. http://www.hybrid.univ-paris8.fr/lodel/index.php?id=287

Becker, K. (2011). The Magic Bullet: A Tool for Assessing and Evaluating Learning Potential in Games. International Journal of Game-Based Learning, 1(1), 19-31.

Cañal, P., Pozuelos, F. J., y Travé, G. (2005). Proyecto Curricular Investigando Nuestro Mundo (612). Descripción General y https://inmweb.wordpress.com/libros/descripcion-general-y-fundamentos/

Cuenca, J. M. (2012). ¿̇Se aprende geografía e historia a través de los videojuegos? En Actas I Congreso Internacional Videojuegos y Educación (pp. 50-57). Departamento de Teoría de la Educación, Facultad de Filosofía y Ciencias de la Educación (Universidad de Valencia). https://www.uv.es/ordvided/ACTAS/ACTAS\%20CIVE\%202012.pdf.

Cuenca, J. M. y Martin, M. (2010). La resolución de problemas en la enseñanza de las Ciencias Sociales a través de los videojuegos. Revista íber, 63, 32-42.

Cuenca, J. M., Martin, M. y Estepa, J. (2011). Historia y videojuegos. Revista Íber, 69, 64-73.

Del Moral, E. (1996). Videojuegos, juegos de rol y simuladores. En Cuadernos de Pedagogía, 246, 84-88.

Del Rincón, D., Arnal, J., La Torre, A., Sans, A. (1995). Técnicas de investigación en Ciencias Sociales. Dyckinson.

Egea Vivancos, A. y Arias-Ferrer, L. (2020). Principles for the design of a history and heritage games based of the evaluation of immersive virtual reality video games. $E$-Learning and Digital Media, 18(4), 383-402. https://doi.org/10.1177/2042753020980103

Egea Vivancos, A., Arias Ferrer, L., y García López, A. J. (2017). Videojuegos, historia y patrimonio: primeros resultados de una investigación educativa evaluativa en educación secundaria. Revista Interuniversitaria de Investigación en Tecnología Educativa, 2, 28-40. https://doi.org/10.6018/riite/2017/283801

Eguia Gómez, J. L., Contreras-Espinosa, R. y Solano-Albajes, L. (2013). Videojuegos: conceptos, historia y su potencial como herramienta para la educación. Ciencias, 1(2), 1-14

Esnaola, G. A. y Levis, D. (2008). La narrativa en los videojuegos: un espacio cultural de aprendizaje socioemocional. Revista Electrónica Teoría de la Educación. Educación y Cultura en la Sociedad de la Información, 9(3), 48-68. https://www.redalyc.org/pdf/2010/201017343004.pdf 
Estepa Giménez, J. (2017). Otra didáctica de la Historia para otra escuela. Servicio de Publicaciones Universidad de Huelva.

Etxeberria Balerdi, F. (2008). Videojuegos, Consumo y Educación. Revista Electrónica Teoría de la Educación. Educación y Cultura en la Sociedad de la Información, 9(3), 11-28. https://www.redalyc.org/pdf/2010/201017343002.pdf

Etxeberria Balerdi, F. (2012). Videojuegos: riesgos y oportunidades en educación. En F. J. Sánchez i Peris, I. Verde Peleato, C. Ros Ros y M. C. Bellver Moreno, Actas I Congreso Internacional Videojuegos y Educación (pp. 22-32). Departamento de Teoría de la Educación, Facultad de Filosofía y Ciencias de la Educación (Universidad de Valencia). https://www.uv.es/ordvided/ACTAS/ACTAS\%20CIVE\%202012.pdf

Gálvez de la Cuesta, M. (2006). Aplicaciones de los videojuegos de contenido histórico en el aula. Revista ICONO 14. Revista Científica De Comunicación Y Tecnologías Emergentes, 4(1), 217-230. https://doi.org/10.7195/ri 14.v4i1.405

Gee, J. P. (2004). Lo que nos enseñan los videojuegos sobre el aprendizaje y el alfabetismo. Aljibe.

Goetz, J. P. y Lecompte, M. D. (1988). Etnografía y diseño cualitativo en investigación educativa. Morata.

Gros, B. (2000). La dimensión socioeducativa de los videojuegos. Edutec. Revista Electrónica de Tecnología Educativa, 12. https://doi.org/10.21556/edutec.2000.12.557

Gros, B. (2012). Estrategias de aprendizaje basado en videojuegos en la formación del profesorado. En Actas I Congreso Internacional Videojuegos y Educación (pp. 5-16). Facultad de Filosofía y Ciencias de la Educación-Universidad de Valencia). https://www.uv.es/ordvided/ACTAS/ACTAS\%20CIVE\%202012.pdf

Grossman, D. (2000). Teaching kids to kill. Phi Kappa Phi National Forum, 80(4), 10. Recuperado de https://www.killology.com/teaching-kids-to-kill

Herrán, A. de la (2005). Los grandes paradigmas científicos. En A. Herrán, E. Hashimoto, y E. Machado. Investigar en Educación: Fundamentos, aplicación y nuevas perspectivas (pp. 25 34). Dilex.

Ibáñez-Etxeberria, A, Asensio, M., Correa, J. (2011). Mobile Learning y Patrimonio: Aprendiendo Historia con mi teléfono, Mi GPS y Mi PDA. En A. Ibáñez-Etxeberria (Ed.), Museos, redes sociales y tecnología 2.0 (pp 59-88). Servicio Editorial de la Universidad del País Vasco

Iturriaga, D. (2015). Ensenar Historia haciendo visible lo invisible a través de los videojuegos de Historia en secundaria. En A. Hernández, C. R. García y J. L. De la Montaña, Una enseñanza de las ciencias sociales para el futuro: recursos para trabajar la invisibilidad de personas, lugares y temáticas. (pp. 217-223). Universidad de Extremadura.

Jiménez-Palacios, R. (2020). Experimentación y análisis del uso de los videojuegos para la educación patrimonial. Un estudio de caso en $1 .^{\circ}$ de ESO. [Tesis Doctoral no publicada]. Universidad de Huelva

Jiménez-Palacios, R. y Cuenca, J. M. (2015). El uso didáctico de los videojuegos. Concepciones e ideas de futuros docentes de ciencias sociales. CLIO. History and history teaching, 41. http://clio.rediris.es/n41/articulos/JimenezCuenca2015.pdf 
Luna, U., Ibañez-Extebarria, A. y Rivero, P. (2019). El Patrimonio aumentado. 8 apps de Realidad Aumentada para la enseñanza-aprendizaje del patrimonio. Revista Interuniversitaria de Formación del Profesorado, 33(1), 43-62.

Martín-Cáceres, M. J. y Cuenca López, J. M. (2019). Evaluar aprendizajes con videojuegos de historia. Iber. Didáctica de las Ciencias Sociales, Geografía e Historia, 95, 30-37.

Martínez Rodríguez, J. (2011). Métodos de investigación cualitativa. Revista Silogismo, 4(8), 2838

Monjelat, N. y Méndez, M. (2012). Videojuegos y diversidad: construyendo una comunidad de práctica en el aula. RED. Revista de Educación a Distancia, 33, 1-23

Muñoz, J. G., (2012). La implementación de los principios científico didácticos (PCD) para la enseñanza de la Geografía y la Historia a través de los videojuegos. En I Congreso Internacional Virtual Innovación Pedagógica y Praxis Educativa (pp. 435-444). Asociación para la Formación, el Ocio y el Entretenimiento. https://dialnet.unirioja.es/servlet/libro?codigo $=535470$

Mugueta, I., Manzano, A., Alonso, P. y Labiano, L. (2015). Videojuegos para aprender Historia: una experiencia con Age of Empires. Revista Didáctica, Innovación y Multimedia, 32. http://dimglobal.net/revistaDIM32/DIMOC32jugarhistoria.htm

Pallarés Piquer, M. (2013). Videojuegos y educación: una combinación al servicio de la mediación entre los aprendizajes de la escuela del siglo xxi y su alumnado. En Actas II Congreso Internacional Videojuegos y Educación (pp. 218-240). Bubok Publishin S.L. https://es.slideshare.net/eraser/libro-cive-13

Provenzo, E. (1991). Video Kids making sense of Nintendo. Harvard University Press. https://doi.org/10.4159/harvard.9780674422483

Sánchez i Peris, F. J. (2012). Introducción: los videojuegos en la educación. En Actas I Congreso Internacional Videojuegos y Educación (pp. 1-4) Departamento de Teoría de la Educación, Facultad de Filosofía y Ciencias de la Educación (Universidad de Valencia). https://www.uv.es/ordvided/ACTAS/ACTAS\%20CIVE\%202012.pdf

Siurot, M. (1964). Cosas de niños. Escuela Gráfica Salesiana.

Venegas, A. (2019). El videojuego histórico como memoria literal y memoria ejemplar. Historiografías, revista de historia y teoría, 18, 30-54. https://papiro.unizar.es/ojs/index.php/historiografias/article/view/4177

Venegas, A. (2020). Las mecánicas como forma de interacción activa con el pasado. Presura. http://www.presura.es/blog/2020/06/09/las-mecanicas-como-forma-de-interaccionactiva-con-el-pasado/ 


\section{Panta Rei}

PANTA REI es una revista digital de investigación orientada a la Historia y otras ciencias afines. Su principal objetivo es la transmisión del conocimiento científico, dando una oportunidad también a los jóvenes investigadores que quieren abrirse camino en el estudio de las ciencias humanas y sociales. Se compone de estudios originales relacionados con la disciplina histórica así como su didáctica y difusión. Las diferentes secciones que componen la revista son: artículos de investigación, entrevistas a profesionales, recensiones de monografías de actualidad y crónicas de congresos o eventos científicos relevantes.

Todos los artículos publicados son objeto de un proceso de revisión a cargo de un mínimo de dos evaluadores, que se consideran expertos en el ámbito temático del artículo propuesto. Nuestro deseo es poder ofrecer unos contenidos rigurosos, de calidad y de interés.

El CEPOAT (Centro de Estudios del Próximo Oriente y la Antigüedad Tardía de la Universidad de Murcia) es la institución encargada de la coordinación y gestión de la revista, desde donde anualmente se lanzará la convocatoria para aquellos que estén interesados en publicar sus trabajos, siempre relacionados con la Historia, Arqueología, Historia del Arte, Didáctica de las Ciencias Sociales, etc.

PANTA REI is a digital journal focused on History and other sciences related to it. Its main objective is the transmission of scientific knowledge by giving also an opportunity to young researchers who want to make their way in the study of human and social sciences. It is composed by original studies related to History, as well as its didactics and promotion. The different sections of this journal are: research articles, interviews to professionals, recensions on monographs about current issues and reports about congresses or relevant scientific events.

All the articles published are subject to a revision process carried out by a minimum of two reviewers who are considered to be experts in the field of the article proposed. Our wish is to offer rigorous contents with quality and being of interest to the reader.

CEPOAT (Centre of Studies of the Middle East and Late Antiquity of the University of Murcia) is the institution in charge of the coordination and management of this journal. This is the centre from where the call for papers will be launched for all the people interested in publishing their papers, always related to History, Archeology, Art History, Didactics of the Social Sciences, etc. 


\section{Normas de publicación}

El autor se compromete a enviar trabajos originales, que no se encuentren publicados en otras revistas ni en otros idiomas. Así mismo, el mismo artículo no podrá ser presentado en otras revistas mientras dure el proceso de evaluación.

\section{Envío y presentación de originales}

Los artículos se enviarán exclusivamente a través del correo electrónico a la dirección pantarei@um.es. Los textos serán enviados en formato DOC y las imágenes en formato JPEG o TIFF, y con un tamaño mínimo de 2000 px. Éstas no aparecerán incorporadas en el texto, sino enviadas en archivo aparte y correctamente numeradas según su posición en el texto. Junto al trabajo, se rellenará y enviará un documento aparte en el que se especifiquen los datos del autor siguiendo el modelo disponible en la página Web de la revista.

Para la redacción de los trabajos se tendrá en cuenta el Manual de la American Psychological Association, en su sexta edición. La extensión máxima de los trabajos será de 30 páginas. La tipografía será Arial 11, con interlineado sencillo y sin espacio alguno entre párrafos. El texto deberá ir justificado a ambos márgenes y sin sangría en los primeros párrafos. Los márgenes serán de 2,50 cm. En los casos en los que fuera necesario incorporar notas, éstas irán a pie de página, enumeradas consecutivamente, con tipografía Arial 10, interlineado sencillo y justificadas a ambos márgenes.

Una información más detallada se encuentra disponible en la página http://www.um.es/cepoat/pantarei.

\section{Proceso de valoración y evaluación}

Una vez recibidos los trabajos, la Revista realizará una primera valoración. Si el trabajo enviado se ajusta a las normas de presentación propuestas, la temática es coincidente con la línea editorial de la revista y posee la calidad científica necesaria, será remitido al consejo asesor para una primera evaluación. Si no es así en este primer paso se puede rechazar directamente los documentos que incumplan claramente la línea editorial.

Será el Consejo Asesor quien indique a la revista la originalidad, relevancia, estructura, redacción, aparato bibliográfico, etc. del trabajo enviado y, para ello, se designará a dos revisores expertos externos que evaluarán cada uno de los trabajos, que pueden formar parte (o no) de este Consejo Asesor. La selección de los revisores se ajustará a la temática y características metodológicas del trabajo. El nombre y filiación de los autores serán eliminados del trabajo para su revisión, así como los revisores actuarán de manera anónima y confidencial.

Los revisores deberán rellenar un informe de evaluación que centrará su atención en aspectos tales como características formales, originalidad y novedad de los trabajos, relevancia de las propuestas y los resultados, calidad metodológica y validez científica.

Una vez terminado el proceso se decidirá la aceptación o no de los mismos y su publicación en el número que sea pertinente, así como las modificaciones susceptibles de ser realizadas para su final publicación. Dicha notificación se enviará únicamente por correo electrónico, en un plazo máximo de seis meses. 


\section{Publishing rules}

The author is committed to submit original papers not having been published in other reviews or in other languages. In this way, it is not allowed for the same paper to be presented in other reviews during the evaluation process.

\section{Submission and presentation of originals}

The articles will be exclusively submitted by email to pantarei@um.es. The texts will be submitted in DOC format and the images in JPEG or TIFF format, and with a minimum size of 2000 px. Images will not be integrated in the text but sent in another file and properly numbered according to their position in the text. Attached to the paper, a document will be filled out and sent where the author's data will be specified following the model available on the website.

The sixth edition of the Manual of the American Psychological Association will be taken into account for the writing of the papers. The length of the papers must not exceed 30 pages. Typography will be Arial 11, with simple line spacing and no space between paragraphs. The text must be justified on both margins without indentation in the first paragraphs. Margins size will be $2.50 \mathrm{~cm}$. Where it could be necessary the incorporation of notes, they will be at the bottom of the page, consecutively numbered with typography Arial 10, simple line spacing and justified on both margins.

More detailed information is available on the website: http://www.um.es/cepoat/pantarei.

\section{Examination and assessment process}

The Journal will submit the papers to a first examination once received. If the paper follows the presentation guidelines, the subject agrees with the editorial line of this journal, and possess the scientific quality required, it will be sent to the advisory council for a first assessment. If not, the documents which clearly fail to complete the editorial line may be rejected straightaway in this first step.

The Advisory Council will indicate the originality, relevance, structure, writing, bibliography, etc. of the text to the journal; for this purpose, two outside experts will be designated to review the papers; these experts can be (or not) part of this Advisory Council. The selection of the experts will adjust to the subject and methodological characteristics of the paper. Name and affiliation of the author will be eliminated from the text for its review, in this way experts will act anonymously and confidentially.

The experts will fill out an assessment report which will focus on aspects such as formal characteristics, originality and novelty of the papers, relevance and results of the proposal, methodological quality and scientific validity.

Once the process is finished, the acceptance or not of the papers and its publication in the corresponding edition will be decided, as well as the modifications that may be done for its final publication. This notification will be sent by email within 6 months maximum. 


\section{cepoAt edit.um}

